Review Article

\title{
Diagnosis and management of ventricular tachycardia
}

\author{
Mark Dancy
}

Central Middlesex Hospital, Acton Lane, London NW10 7NS, UK

\begin{abstract}
Introduction
Ventricular tachycardias are a diverse group of arrhythmias with widely differing properties and outcomes. This article will concentrate on those due to structural and, in particular, ischaemic heart disease as they are by far the commonest and most dangerous.
\end{abstract}

\section{Diagnosis of ventricular tachycardia}

Thirty per cent of all patients with ventricular tachycardia (VT) die within 12 months of diagnosis. In those with severe structural heart disease the mortality in the first year is around $80 \%{ }^{1}$ Appropriate treatment considerably reduces mortality and can only be given if the condition is correctly diagnosed.

In current medical practice there can be few commonly occurring conditions which are surrounded by so much misunderstanding and preconception. Recent studies show that there is approximately a $\mathbf{4 0} \%$ error rate in diagnosing broad complex tachycardia ${ }^{2}$ whereas correct analysis of the electrocardiogram (ECG) features alone should allow the diagnosis of VT to be made correctly in over $90 \%$ of cases. ${ }^{3}$ The errors are in part due to a poor understanding of the electrocardiographic criteria for distinguishing VT from supraventricular tachycardia (SVT), particularly as far as the reliability of individual indicators is concerned.

All grades of hospital doctor confuse VT with SVT. Usually the mistake is to diagnose SVT when the rhythm is in fact VT. ${ }^{3}$ The possible reasons and motives for this bias are fascinating. It is almost as if doctors feel more comfortable with SVT and that they are so unwilling to get involved in the complexities of the management of VT, that they 'prefer' to make the diagnosis of SVT rather than VT. Misdiagnosis may be a consequence of common

Correspondence: M. Dancy, M.R.C.P.

Accepted: 7 January 1992 misconceptions including the idea that VT always causes severe haemodynamic disturbance. In fact patients often present with minor symptoms such as palpitations. ${ }^{4}$ It may be a failure to recognize that VT is more common than SVT as a cause of broad complex tachycardia ${ }^{5}$ and that broad complex tachycardia in patients with ischaemic heart disease is almost invariably VT. ${ }^{6}$ There is still a misconception that verapamil is safe, whereas the studies suggest that it causes circulatory collapse in such a high proportion of patients ${ }^{7}$ that it could be regarded as negligent to use it in patients with broad complex tachycardias, unless the diagnosis of SVT were to be firmly established. There may b a feeling that the subtleties of electrocardiographie interpretation are the province of electrophysio logists and that the simple practising clinician? might as well toss a coin as spend time comparing ECGs. This complete lack of self-confidence is typified by the ready acceptance of previously made diagnoses ('known SVT'). ${ }^{8}$

From the practical point of view there are two phases to the diagnosis of ventricular tachycardia. The first is the initial presentation. The patient may either be extremely ill or virtually asymptomatic but the only information available is from the history, clinical findings and the ECG during tachycardia. Here, sometimes decisions have to be made rapidly with less than full information and it may not be possible to diagnose the origin of the arrhythmia accurately. Then it may be sufficient to call the arrhythmia 'broad complex tachycardia' provided that the potential mechanisms are understood and that the treatment given is appropriate to all the potential causes. Following the restoration of sinus rhythm, there is much more electrocardiographic information and the process of planning long-term management begins. In this second phase, the label broad complex tachycardia is unacceptable as it gives no idea of prognosis and no grounds on which to choose therapeutic agents.

Because all forms of ventricular tachycardia (with the exception of the rare fascicular tachycardia) produce complexes of $120 \mathrm{~ms}$ or more, it is usually the presence of broad complexes that first 
raises the possibility of ventricular tachycardia. Apart from VT the possible alternative causes of broad complex tachycardia are: (1) supraventricular tachycardia with bundle branch block caused by temporary inability of part of the conducting system to conduct at a fast rate; (2) supraventricular tachycardia arising in a patient with preexisting bundle branch block; or (3) tachycardias conducted through an accessory pathway (either re-entrant or atrially driven). These three groups can be distinguished from VT with the help of the 12-lead ECG in the majority of cases.

Over the last 20 years there have been numerous attempts to produce reliable criteria for the diagnosis of VT by surface ECG. The fact that misdiagnosis is still common testifies in part to doctors' ignorance of the available criteria but also suggests that the criteria are imperfect. One problem is that there has been little attempt until recently to categorize the criteria into those that have high sensitivity and specificity and those whose predictive power is low. There is little point in memorizing a complex QRS morphology if it is only weakly predictive of VT. Furthermore, some of the criteria which were originally believed to be valuable have subsequently been shown to be potentially misleading. ${ }^{9}$ Two recent studies have attempted to address this problem ${ }^{9,10}$ by concentrating on those criteria with the greatest predictive power for VT. Combining results from these studies, the features are listed in Table $\mathrm{I}$.

Absence of RS complex in every precordial lead is a newly described criterion in one recent study where it was found that, out of 554 ECGs showing broad complex tachycardia, all the 170 supraventricular tachycardias had at least one precordial lead showing an RS complex. Therefore absence of an RS complex was $100 \%$ specific for the diagnosis of VT. ${ }^{9}$ This criterion has yet to be corroborated but appears to be a very simple and accurate method for diagnosing a proportion of VTs. Some
VTs did show an RS in one of the precordial leads so if there is an RS it does not denote SVT.

Atrioventricular dissociation can be manifest in a number of ways and is particularly important because it rules out an atrial origin for the tachycardia and is therefore the most powerful diagnostic feature. By definition it is always present in VT but it is not always apparent on the surface ECG either because the $P$ waves may be too insignificant in relation to the large QRS complexes or because there may be retrograde conduction of every ventricular cycle to the atria (1:1 ventriculo-atrial (VA) conduction) producing one $P$ wave for every QRS and confusingly suggesting a supraventricular origin.

Patterns of atrioventricular dissociation which may be seen (but are often unnoticed) are an atrial rate that is completely different from the ventricular rate. This may give rise to capture beats, where atrial depolarization happens to occur at a time when the ventricles are excitable (giving rise to a sudden sinus beat with narrow QRS complex), or fusion beats, where there is almost simultaneous excitation of the ventricles from the ventricular ectopic focus and from the sinus node via the atrio-ventricular node (giving rise to a QRS complex which may be any shape from broad to narrow depending on the electrical circumstances). There may also be partially blocked VA conduction where there is a highly characteristic pattern of $P$ waves appearing every alternate, third, fourth, fifth, etc. QRS complex. This is very easy to spot if systematically looked for because of the absence of $\mathbf{P}$ waves in the intervening cycle/cycles.

QRS morphology may be very valuable in distinguishing SVT from VT but unfortunately the patterns are more difficult to memorize. The most valuable indicators of VT are: (a) concordance, where the precordial leads are either all positive or all negative; (b) $R s^{1}$ in V1 (i.e. the initial ' $R$ ' is larger than the secondary ' $r$ ') or (c) deep monophasic

Table I Predictive power and prevalence of features in ventricular tachycardia

\begin{tabular}{lcc}
\hline Feature & $\begin{array}{c}\text { Positive } \\
\text { predictive } \\
\text { power }\end{array}$ & $\begin{array}{c}\text { \% of all VT } \\
\text { showing } \\
\text { feature }\end{array}$ \\
\hline Atrioventricular dissociation (A-V) dissociation & 1.0 & 10 \\
Absence of RS complex in every precordial lead & 1.0 & 15 \\
R-S interval $>100$ ms in any precordial lead & 0.98 & 32 \\
History of myocardial infarct & 0.97 & 44 \\
History of angina & 0.96 & 22 \\
Ventricular extrasystoles with same morphology in sinus rhythm & 1.0 & 12 \\
Cardiothoracic ratio $>0.55$ & 0.97 & 33 \\
Known left ventricular aneurysm & 1.0 & 14 \\
Concordance & 1.0 & 26 \\
Detailed morphological analysis & 0.96 & - \\
\hline
\end{tabular}


QS wave in V6.

Because bundle branch block patterns are consistent, any two episodes of broad complex tachycardia which are due to SVT with bundle branch block should look the same. By contrast some patients with VT display different surface ECG patterns on separate occasions. When two or more different morphologies of broad complex tachycardia are documented on different occasions in the same patient it is likely that both are forms of VT.

\section{Treatment of the acute event}

The choice of treatment for patients admitted with broad complex tachycardia depends on their haemodynamic status. Any unconscious patient should be DC cardioverted immediately. A patient in shock should have a 12-lead ECG recorded and then be cardioverted.

For the more stable patient there is more time and it should be used for analysis of the ECG on the lines discussed above, a detailed history including previous ischaemic events, tachycardias and current and past drug therapy. If possible any old case notes should be retrieved to see if there are any previous ECGs of either sinus rhythm or tachycardia. If there is still doubt as to the origin of the tachycardia it may be helpful to record an oesophageal or intra-atrial ECG during tachycardia to demonstrate $\mathrm{A}-\mathrm{V}$ dissociation that was not apparent on the surface ECG.

In this more stable group of patients it is valuable to attempt to restore sinus rhythm by intravenous antiarrhythmic drug treatment because, if it successfully terminates the tachycardia, the chosen drug may be valuable when given orally to prevent subsequent attacks. Indeed it may be the only opportunity one has to test a specific drug against the spontaneously occurring tachycardia. Only one drug should be used and, if it is unsuccessful in terminating VT, DC cardioversion should follow. If there is a possibility of drug toxicity, such as with digoxin, overdrive pacing may be safer than DC shock.

Once sinus rhythm is restored the 12-lead ECG should be repeated and all the information about the event reviewed in an attempt to arrive at a firm retrospective diagnosis. The principles discussed above may be systematically applied and algorithms may be helpful. ${ }^{11}$ Where there remains doubt as to the the mechanism of the arrhythmia, particularly in patients who are potentially at significant risk if the arrhythmia is VT (vide infra), then there is a case for attempting to provoke it under controlled conditions using electrophysiological study (EPS) when the mechanism can be fully documented.

\section{Long-term management of VT}

Once a patient is back in sinus rhythm following an episode of ventricular tachycardia, there are a number of choices of action, as listed in Table II

The goal of treatment is the elimination of the arrhythmia, or at least the reduction of the risk associated with its recurrence. The chosen agent must not introduce a new risk, or, if it does, that risk must be less than that of the original arrhythmia. In other words the risk-benefit ratio must improve with the introduction of the treatment.

\section{The risk of VT}

Although it is not possible to define risk at all accurately, the following broad statements appear to be true. (1) The risk of non-sustained VT is less than that of sustained VT. ${ }^{12,13}$ (2) The risk of VT (either sustained ${ }^{14}$ or non-sustained ${ }^{15,16}$ ) increases in proportion to the degree of structural heart disease (usually expressed as ejection fraction - the lower the ejection fraction the higher the risk). ${ }^{17}$ Even simple clinical grading of dyspnoea is a good predictor of risk. ${ }^{18}$ Patients with clinically normal hearts have an excellent prognosis even if detailed evaluation shows up minor abnormalities. ${ }^{19}(3)$ The more severe the symptoms caused by the VT the greater the subsequent risk. This is a complex observation related to left ventricular function and the rate and stability of VT. ${ }^{20}$ Cardiac arrest during the index arrhythmia is associated with a particularly high risk for subsequent events. ${ }^{14}$ (4) Because the risks of non-sustained VT are less than sustained VT it is more difficult to improve the risk-benefit ratio with drug therapy. Patients with non-sustained VT should not be treated unless they are shown to be at risk (previous cardiac arrest or other significant symptom, severe left ventricular disease), and only then if the chosen drug can be shown to be effective. ${ }^{21}$

Table II Choices for long-term management of ventricular tachycardia

1. Do nothing.

2. Remove a potential cause (drug, electrolyte disturbance, ischaemia).

3. Treat with antiarrhythmic drug.

4. Manage with implantable cardioverter defibrillator.

5. Disrupt the structure of the arrhythmogenic area in the ventricle.

6. Cardiac transplantation for a highly select group of patients whose rhythm cannot be managed in any other way and who are considered to be at high risk. 


\section{Potential benefits of treatment}

\section{(a) Concomitant drug treatment}

In patients thought to be at risk of ventricular arrhythmias, close attention needs to be given to any drugs that have been prescribed for other purposes, particularly for the treatment of heart failure and atrial fibrillation. Inappropriate or unbalanced use of diuretics may increase the risk of arrhythmia by the induction of hypokalaemia or hypomagnesaemia. The overall risk-benefit balance with digoxin (frequently used in the treatment of atrial fibrillation in heart failure, and less often used for the treatment of heart failure in sinus rhythm) is probably neutral ${ }^{22}$ provided of course that the dosage is not excessive. Angiotensin converting enzyme (ACE) inhibitors do however have mild antiarrhythmic effects, reducing the frequency of ventricular ectopy and more complex forms. ${ }^{23}$ This effect may be causally related to the fact that ACE inhibitors improve survival in moderate and severe heart failure. ${ }^{24,25}$ As the indications for ACE inhibition become broader, so the sort of patients who suffer from ventricular arrhythmias are increasingly likely to be treated with ACE inhibitors.

\section{(b) Antiarrhythmic drug treatment}

The ideal antiarrhythmic drug abolishes the index arrhythmia without introducing new risks. This is an easy enough principle to state but in practice it is more difficult to demonstrate that a particular drug is effective and likely to remain so in the future. How can one be assured that a patient who had been given a drug for a length of time would have had a spontaneous arrhythmic event had it not been for the treatment he had been given? This question would be easier to answer if there was better understanding of one of the fundamental questions in arrhythmia physiology - why, in a susceptible individual, does a particular episode of arrhythmia start when it does, whereas 5 minutes beforehand and 2 hours later the patient is in stable sinus rhythm?

It seems likely that for any arrhythmia to develop there must be a conjunction of factors that are specific to that arrhythmia. There must be a potential 'electrical circuit' but for the circuit to express itself there must usually be some triggering factor or factors. Some of these triggers are well recognized (ventricular or atrial ectopics, hypokalaemia, ${ }^{26}$ hypertrophy ${ }^{27,28}$ and ischaemia, for example), but often in the individual patient the known triggers do not fully explain every episode of spontaneous arrhythmia, ${ }^{29}$ suggesting there are other unidentified triggers. It is very likely that the autonomic nervous system ${ }^{30-32}$ is of great impor- tance in this area, but the mechanisms are incompletely understood.

To test whether a drug is effective one would ideally recreate the exact conditions that led to the arrhythmia developing spontaneously. Because of the lack of understanding of trigger mechanisms, this is usually not possible and artificial methods of provoking arrhythmias, such electrophysiologic study (EPS) may have to be used.

The available tests of efficacy may be divided into invasive and non-invasive, provocative and non-provocative. Non-invasive and non-provocative include symptom relief and bedside or Holter monitoring. Provocative tests are exercise testing and EPS.

If VT is recurring frequently, provocation testing may not be necessary to demonstrate whether a drug is effective, but in the more usual situation, the frequency of spontaneous VT is such that some form of provocation is necessary if only to reduce the time required to be confident that the treatment is protective. ${ }^{33}$ Drug therapy guided by noninvasive testing can undoubtedly be effective if the protocol is carefully designed and strictly adhered to $^{34}$ but there are no reliable studies comparing this approach to the more invasive stimulation techniques. The ESVEM trial (electrophysiologic study versus electrocardiographic monitoring ${ }^{35}$ ) which is due to be completed in 1991 should answer some of the questions but it seems likely that both methods will be shown to have their place ${ }^{36}$ and until EPS is more widely available in the UK the comparison may be largely academic: many hospitals will have to rely on Holter-guided therapy for lack of electrophysiological facilities.

EPS is not wholly reliable and even in controlled laboratory conditions its reproducibility is imperfect. ${ }^{37,38}$ Drugs that are found to be effective with provocation testing are not always effective longterm $^{39}$ when the underlying disease process may well have progressed. ${ }^{40}$ Provocation by exercise testing is probably almost as reproducible and more physiological, ${ }^{41}$ but only in those $20 \%$ of patients whose arrhythmia is reproducibly induced by exercise before the introduction of drug. ${ }^{42}$

Despite the apparent limitations of programmed stimulation numerous studies have demonstrated a number of facts. One is that patients with inducible sustained VT are at higher risk than those in whom VT is not inducible. ${ }^{43,44}$ Another is that in those patients with inducible sustained VT a drug which suppresses it in the electrophysiology laboratory will reduce the subsequent risk of sudden death provided it does not also have proarrhythmic effect at the time of study. ${ }^{1}$ Also failure to find a drug that will suppress inducible VT is associated with a significantly higher mortality. ${ }^{1,45} \mathrm{Up}$ to $50 \%$ of patients may remain inducible after a number of drug trials and these patient are at high risk. ${ }^{46}$ 
This suggests either that drug testing does identify successful drugs or that the process of drug testing identifies a group of patients with a better prognosis (regardless of the treatment) ${ }^{47}$ It is likely that the drugs are responsible for the improvement in prognosis as there is a high reported incidence of arrhythmic events among patients who initially respond to a drug but later default from treatment. ${ }^{1}$

In general the more aggressive the attempts to provoke VT the more likely it is to be provoked, but with very unphysiological stimulation protocols, the VT so initiated may not be the same as the spontaneously occurring VT (as judged by comparison of the surface ECGs). Attempts to prevent the initiation of an 'unnatural VT' may not predict the correct treatment of the spontaneously occurring VT.

\section{Potential risks of drug treatment}

\section{Proarrhythmia}

In patients with ischaemic heart disease the setting for VT is thought to be the juxtaposition of islands of ischaemic or fibrous tissue and the surrounding normal myocardial cells. Under these conditions conduction in the damaged area may be sufficiently slowed that the refractory period of the surrounding normal areas has been completed by the time the wave of excitation has spread through the damaged area. This then creates a perpetual circuit due to re-entry. Drugs may modify the circuit by increasing the refractory period of normal tissue, but many antiarrhythmic agents also slow conduction and thereby may facilitate other circuits which can be as dangerous or more dangerous than the circuit which has been abolished. Usually, because they are other circuits, they will be represented by surface ECG morphologies that are distinct from those of the original tachycardia and they can therefore be recognized as being due to a proarrhythmic effect.

The phenomenon of proarrhythmia encompasses the expression of other circuits as described above and also the worsening of spontaneous arrhythmias (for example, a ventricular tachycardia which has previously been non-sustained may become sustained) under the influence of an antiarrhythmic drug. Although it has long been recognized as a potential problem particularly of Class I agents, it has only recently, with the publication of the CAST study, ${ }^{48}$ been appreciated that it is sufficiently common seriously to influence the risk-benefit ratio even to the extent of causing overall harm, particularly in a population with a relatively low risk before treatment. Even in high risk groups empirical drug treatment may reduce survival. ${ }^{49}$

It is quite clear now that some drugs or groups of drugs are more likely to produce proarrhythmic effects than others. The main culprits are the Vaughan-Williams classes Ia and class Ic (especially flecainide and encainide which were particularly implicated in the CAST study). At the opposite end of the proarrhythmic spectrum are the class II drugs ( $\beta$-blockers). ${ }^{50}$ Despite their frequent proarrhythmic effects, Ia and Ic drugs are still extensively used because they are often highly effective at treating the target VT but, because of the potential for proarrhythmia, a careful search is necessary for evidence of proarrhythmia as well as for evidence that the drug is beneficial in terms of suppressing the original VT. ${ }^{49}$ It may not be quite so important to demonstrate that the antiarrhythmic agent works in patients who are treated with the less proarrhythmic agents such as amiodarone. The BASIS study in survivors of acute myocardial infarction (which may be a rather different group from the patients with long-standing ischaemic heart disease or dilated cardiomyopathy) demonstrated at least as good survival in patients treated with amiodarone empirically as in those whose drug treatment was designed on an individual basis. ${ }^{51}$

Major difficulties arise in demonstrating (or even defining) proarrhythmia in a way that allows scientific analysis. Exactly as with untreated arrhythmias, proarrhythmic effects may be demon strated when they arise spontaneously or followingo some form of deliberate provocation. Eighty per? cent of spontaneously occurring proarrhythmic events occur within 6 days of starting a new drug. ${ }^{52}$ When patients develop VT while on medication there may sometimes be difficulty in deciding whether the VT represents a proarrhythmic effect or the breakthrough of the old arrhythmia. However, whatever the mechanism, the observation of an important spontaneous arrhythmia occurring whilst on treatment will at least cast some doubt on the wisdom of using that particular drug.

Greater difficulties arise when proarrhythmic effects can only be demonstrated by provocation. Non-invasive provocation, of which exercise testing is the commonest form, demonstrates only about half as many episodes of proarrhythmia as more aggressive invasive electrophysiological studies. Yet it is far from clear what is the prognostic implication of proarrhythmia as revealed by EPS or exercise testing. Exercise testing has the major theoretical advantage that it is both physiological and natural, but if exercise (or any resultant myocardial ischaemia) does not happen to be an important trigger for the iatrogenic arrhythmia then clearly a negative exercise test result should not provide much reassurance.

Just as the incidence and risk of spontaneous VT increases with the severity of structural heart disease, so does the incidence and risk of proarr- 
hythmia, particularly in patients taking digitalis and diuretics as well. ${ }^{53,54}$ It is these patients with badly damaged hearts that particularly need the most thorough individual assessment of the riskbenefit ratio, and this implies invasive provocation studies.

\section{Which drugs?}

There is no universally accepted protocol for the drug treatment of VT but some basic concepts are valuable. (1) All antiarrhythmic drugs are potentially proarrhythmic, although $\beta$-blockers are probably the least culpable in this respect. ${ }^{50,55,56}(2)$ All antiarrhythmic drugs with the exception of amiodarone in conventional doses are negatively inotropic, and $\beta$-blockers are the worst (3) $\beta$ blockers either with class III activity (sotalol ${ }^{57,58}$ ) or without (propranolol ${ }^{59}$ ) are being increasingly used in the treatment of VT, although their role may be greatest in catecholamine-sensitive $\mathrm{VT}^{60}$ (e.g. exercise induced or induced by isoprenaline) rather than in the commonest forms of VT following myocardial infarction. ${ }^{61}$ Combination therapy with class I drugs is an exciting area of investiga$\operatorname{tion}^{62}$ as there is a suggestion that $\beta$-blockers may control the proarrhythmic effects of class I agents. ${ }^{63}$ (4) The only drugs that have shown an improvement in survival when used empirically are $\beta$ blockers ${ }^{64}$ and amiodarone ${ }^{51}$ and they have only shown a beneficial effect in the first year after myocardial infarction.

\section{Non-drug management}

Patients at high risk who do not respond to drugs during arrhythmia suppression trials form an obvious group where alternative management needs to be considered. Similarly those who demonstrate proarrhythmic effects with more than one drug may be as much at risk as the non-responder. There are now two alternative approaches. Firstly, there are direct approaches to modify the structure of the arrhythmic circuit. Secondly, the implantable cardioverter defibrillator will electrically pace or cardiovert sustained VT or ventricular fibrillation to sinus rhythm.

\section{(a) Disruption of the structure of the arrhythmogenic focus}

Disruption of the electrical circuit responsible for VT can be achieved by a number of methods which all have the potential to effect a cure.

The site of origin of the tachycardia can be located by mapping either percutaneously or at the time of open heart surgery. In either case the mapping procedure is carried out during induced VT and the most suitable patients therefore must have (a) haemodynamically stable VT to allow time for mapping and (b) monomorphic VT which suggests there is only one circuit to be tackled. Once the area responsible for the VT has been located the tissue can be destroyed by electrical current, cryosurgery or, more recently, radiofrequency discharge. Transvenous catheter ablation has the great potential advantage that it avoids surgery.

A more common surgical procedure is endocardial resection, ${ }^{65}$ where as much damaged endocardium as possible is removed without the necessity of mapping. It has the advantage that other potential arrhythmogenic foci may be removed at the same time. It is now usually combined with map-guided ablation which improves the outcome of the procedure. ${ }^{66}$

All these methods can cure VT. Even in cases where the VT is not cured it is frequently possible to achieve drug suppression of VT where this was not possible prior to ablation/resection. Primary success (no VT recurrence without drugs) is achieved in $18-50 \%$ by transvenous ablation, ${ }^{67-69}$ and $70-$ $100 \%$ by endocardial resection. ${ }^{66,70}$ A further $15-20 \%$ can be successfully controlled by drugs after the procedure whereas previously drugs were ineffective. Five-year survival after surgery is around $60 \%$, the main cause of death being heart failure. The resuts of transvenous ablation for VT are at present very poor and in marked contrast to the results of ablation for supraventricular arrhythmias $^{67}$ which probably reflects the complexity of the substrates responsible for ischaemic VT.

A surprisingly high mortality of $7 \%$ has been reported for transvenous catheter ablation of VT. ${ }^{68}$ This is much higher than that due to other ablation procedures ${ }^{67}$ and probably reflects the seriousness of the underlying disease as well as the problems of high energy shocks in the left ventricle.

Surgical ablation/endocardial resection has a relatively high operative mortality of around $15 \%$, as many patients have very poor left ventricular function and can ill afford the loss of any functioning myocardium. Patients at particularly high risk are those with poor left ventricular function, polymorphic VT, diffuse myocardial damage ('ischaemic cardiomyopathy' rather than a resectable scar or aneurysm) and previous cardiac surgery. ${ }^{71}$

\section{(b) The implantable cardioverter defibrillator}

This device is capable of recognizing VT or ventricular fibrillation and converting it to sinus rhythm. Rapid changes in technology have already ironed out some of the problems with the early devices and current models are capable of delivering 100-150 shocks with relatively little distur- 
bance to the patient. Further improvements now being evaluated include a completely transvenous system and the incorporation of antitachycardia pacing facilities which will enable many arrhythmias to be terminated without the need for high energy discharges and thereby increasing the longevity of the units. The implantable cardioverter defibrillator (ICD) can be used in conjunction with any of the treatment modalities already discussed. ${ }^{72}$

The ICD has proved highly successful in the management of VT, with an incidence of sudden death after implantation as low as $2 \%$ in the first year and $7 \%$ at 4 years. ${ }^{73}$ It can be used in patients for whom no other method is practicable or in those for whom other methods have been unsuccessful.

The risks of death due to implantation using a thoracotomy are surprisingly high in some series (up to $4 \%$ ) but this may be reduced by transvenous systems. Many other complications similar to but more frequent than those experienced in standard pacemaker implantation (infection, lead displacement, device malfunction, etc.) have all been reported. Inappropriate discharge of the devices have occurred in $40-50 \%$ of all patients. Such problems are likely to improve rapidly with advances in technology.

Current ICD systems cost about $£ 12,000$ to $£ 15,000$ and the UK Department of Health has yet to agree appropriate funding for them which places unnecessary and unfair strains on the patients and the staff caring for them.

\section{Conclusions}

Drug treatment remains the first goal for the management of sustained VT, but it has become very important to examine critically the evidence that a particular regimen is effective and that it does not produce new arrhythmias. Where VT is recurring frequently it may be sufficient to rely on

\section{References}

1. Swerdlow, C.D., Winkle, R.A. \& Mason, J.W. Determinants of survival in patients with ventricular tachyarrythmias. $N$ Engl J Med 1983, 308, 1436-1442.

2. Stewart, R.B., Bardy, G.H. \& Greene, L. Wide complex tachycardia: misdiagnosis and outcome after emergent therapy. Ann Int Med 1986, 104: 766-771.

3. Dancy, M., Camm, A.J. \& Ward, D. Misdiagnosis of chronic recurrent ventricular tachycardia. Lancet 1985, ii: 320-323.

4. Wellens, H.J.J. The wide QRS tachycardia. Ann Int Med 1986, 104: 879.

5. Steinman, R.T., Herrera, C., Schuger, C.D. \& Lehman, M.H. Wide QRS tachycardia in the conscious adult: ventricular tachycardia is the most common cause. JAMA 1989, 261: 1013-1016.

6. Tchou, P., Young, P., Mahmud, R., Denker, S., Jazayeri, M. \& Akhtar, M. Useful clinical criteria for the diagnosis of ventricular tachycardia. Am J Med 1987, 84: 53-56. non-invasive methods of assessing response to treatment, but in most instances the quickest way to arrive at the conclusion that a drug regimen is effective and without proarrhythmic effects is by EPS. Simple VT stimulation equipment is not expensive and any cardiologist trained in invasive techniques should be able to master a suitable protocol even in a district general hospital. Indeed until VT stimulation is more widely practised patients will continue to be treated empirically because of the limited number of specialist centres able to carry out antiarrhythmic drug testing.

Patients at high risk are those with significant myocardial damage. If a suitable drug or simple drug combination cannot be found then the choice lies between an ICD and ablation/resection. Factors favouring surgery would be: (1) frequent VT which would rapidly deplete an ICD; $(2)$ easily inducible VT with one or two morphologies at most; (3) associated reversible ischaemia for which surgery was contemplated; (4) ventricular aneursym (particularly anterior); and (5) patient preference. ICD patch electrodes can be inserted at the time of surgery to facilitate the subsequent implantation of an ICD if it becomes necessary.

Factors favouring an ICD would be: (1) infrequent episodes; (2) very poor left ventricular function; (3) partial success with drug therapy; (4)尺 multiple morphologies of VT; (5) no other cardiace surgery required; and (6) patient preference.

Recommendations for non-sustained VT are? very much more difficult to make because prediction of risk is less precise. The most difficult decision is whether to treat at all, and this has to be based on the clinical presentation (syncope, cardiac arrest, palpitations or chance finding) and the degree of underlying heart disease (ejection fraction or equivalent). Of the various treatment modalities, the ICD is the least appropriate and ablative methods may be frustrated by difficulties in mapping. For these reasons drugs are the commonest method of treatment.

7. Rankin, A.C., Rae, A.P. \& Cobbe, S.M. Misuse of intravenous verapamil in patients with ventricular tachycardia. Lancet 1987, ii: 472-474.

8. Anon. 'Looks like SVT'. Lancet 1986, ii: 612-613.

9. Brugada, P., Brugada, J., Mont, L., Smeets, J. \& Andries, E.W. A new approach to the differential diagnosis of a regular tachycardia with a wide QRS complex. Circulation 1991, 83: 1649-1659.

10. Griffiths, M.J., DeBelder, M.A., Linker, N.J., Ward, D.E. \& Camm, A.J. Multivariate anlaysis to simplify the differential diagnosis of broad complex tachycardia. Br Heart J 1991, 66: 166-174.

11. Dancy, M. \& Ward, D. Diagnosis of ventricular tachycardia: a clinical algorithm. Br Med J 1985, 291: 1036. 
12. Pratt, C.M., Francis, M., Mahler, S., Aogaichi, K., Keus, P. \& Young, J.B. A natural history of benign and potentially malignant ventricular arrhythmias with special reference to nonsustained ventricular tachycardia. Am Heart J 1988, 116: 897-902.

13. Follansbee, W.P., Michelson, E.L. \& Morganroth, J. Nonsustained ventricular tachycardia in ambulatory patients: characteristics and association with sudden cardiac death Ann Intern Med 1980, 92: 741-747.

14. Willems, A.R., Tijssen, J.G.P., VanCapelle, F.J. et al. Determinants of prognosis in symptomatic ventricular tachycardia or ventricular fibrillation late after myocardial infarction. JACC 1990, 16: 521-530.

15. Gomes, J.A., Winters, S.L., Stewart, D., Horowitz, J.A., Milner, M. \& Barreca, P. A new non-invasive index to predict sustained ventricular tachycardia and sudden death in the first year after myocardial infarction based on signal averaged electrocardiogram, radionuclide ejection fraction and Holter monitoring. JACC 1987, 10: 349-353.

16. Veltri, E.P., Platia, E.V., Griffith, L.S.C. \& Reid, P.R. Programmed electrical stimulation and long-term follow-up in asymptomatic, non-sustained ventricular tachycardia. $\mathrm{Am}$ $J$ Cardiol 1985, 56: 309-314.

17. Weaver, W.D., Lorch, G.S., Alvares, H.A. \& Cobb, L.A Angiographic findings and prognostic indicators in patients resuscitated from sudden cardiac death. Circulation 1976, 54: $895-900$

18. Brugada, P., Talajic, M., Smeets, J., Mulleneers, R. \& Wellens, H.J.J. The value of the clinical history to assess prognosis of patients with ventricular tachycardia or ventricular fibrillation after myocardial infarction. Eur Heart $J$ 1989, 10: 747-752.

19. Lemergy, R., Brugada, P., Della Bella, P., Dugernier, T., VandenDool, A. \& Wellens, H.J.J. Nonischaemic ventricular tachycardia. Clinical course and long-term follow-up in patients without clinically overt heart disease. Circulation 1989, 79: 990-999.

20. Saxon, L.A., Uretz, E.F. \& Denes, P. Significance of the clinical presentation in ventricular tachycardia/fibrillation. Am Heart J 1989, 118: 695-701.

21. Mintz, R., Marinchak, R.A., Rials, S.J. \& Kowey, P.R. Clinical management of patients with nonsustained ventricular tachycardia: What do we really know? Cardiology 1990 , 77: $166-180$

22. Gradman, A.H., Cunningham, M., Harbison, M.A., Berger, H.J. \& Zaret, B.L. Effects of oral digoxin on ventricular ectopy and its relation to left ventricular function. $\mathrm{Am} J$ Cardiol 1983, 51: 765-769.

23. Pomini, G., Gribaldo, R., Rugna, A., Lupia, M., Molfese, G. \& Carenza, P. Reduction of complex ventricular arrhythmias after enalapril treatment in patients with advanced stable heart failure. G Ital Cardiol 1991, 21: 59-65.

24. The CONSENSUS Trial Study Group. Effects of enalopril on mortality in severe congestive heart failure. Results of the Cooperative North Scandinavian Enalopril Survival Study (CONSENSUS). $N$ Engl J Med 1987, 316: 1429-1435.

25. Dennick, L.G., Maskin, C.S., Meyer, J.H., Schotz, W.E., Brown, B.W. \& Zatuchni, J. N Engl J Med 1987, 17: 1350 (letter).

26. Dargie, H.J., Cleland, J.G.F., Leckie, B.J., Inglis, C.G., East, B.W. \& Ford, I. Relation of arrhythmias and electrolyte abnormalities to survival in patients with severe chronic heart failure. Circulation 1987, 75 (Suppl IV): 98-107.

27. Anderson, K.P. Sudden death, hypertension and hypertrophy. J Cardiovasc Pharmacol 1984, 6 (Suppl III): III498-III-503.

28. Ghali, J.K., Kadakia, S., Cooper, R.S. \& Liao, Y.L. Impact of left ventricular hypertrophy on ventricular arrhythmias in the absence of coronary artery disease. JACC 1991, 17: $1277-1282$.

29. Meissner, M.D., Akhtar, M. \& Lehmann, M.H. Nonischaemic sudden tachyarrhythmic death in atherosclerotic heart disease. Circulation 1991, 84: 905-912.
30. Kolman, B.S., Verrier, R.L. \& Lown, B. The effect of vagus nerve stimulation upon vulnerability of the canine ventricle: role of sympathetic-parasympathetic interactions. Circulation 1975, 52: 578-585.

31. Morady, F., Nelson, S.D., Kou, W.H. et al. Electrophysiological effects of epinephrine in humans. JACC 1988, 11: 1235-1244.

32. Leclercq, J.F., Maisonblanche, P., Cauchemez, B.\& Coumel, $P$. Respective role of sympathetic tone and of cardiac pauses in the genesis of 62 cases of ventricular fibrillation recorded during Holter monitoring. Eur Heart J 1988, 9: 1276-1283.

33. Bigger, J.T. \& Reiffel, J.A. Holter versus electrophysiologic studies in the management of malignant ventricular arrhythmias. Am J Cardiol 1983, 51: 1464-1465.

34. Graboys, T.B., Almeida, E.C. \& Lown, B. Recurrence of malignant ventricular arrhythmia after antiarrhythmic drug withdrawal. Am J Cardiol 1986, 58: 59-62.

35. The ESVEM Investigators. The ESVEM trial: Electrophysiologic study versus electrocardiographic monitoring for selection of antiarrthythmic therapy of ventricular tachyarrhythmias. Circulation 1989, 79: 1354-1360.

36. Kim, S.G. Values and limitations of programmed stimulation and ambulatory monitoring in the management of ventricular tachycardia. Am J Cardiol 1988, 62: 7I-12I.

37. Beckman, K.J., Velasco, C.E., Krapfchek, J., Lin, H.J., Magro, S.A. \& Wyndman, C.R.C. Significant variability in the mode of ventricular tachycardia induction and its implications for the interpretation of acute drug testing. Am Heart $J$ 1988, 116: 718-726.

38. Kudenchuk, P.J., Kron, J., Walance, C.G., Cutler, J.E., Griffith, K.K. \& McAnulty, J.H. Day-to-day reproducibility of antiarrthymic drug trials using programmed extrastimulus techniques for ventricular tachyarrhythmias associated with coronary artery disease. Am J Cardiol 1990, 66: 725-730.

39. Cobb, L.A., Baum, R.S., Alvares, H.I. \& Schafer, W.A. Resuscitation from out of hospital ventricular fibrillation: four years follow-up. Circulation 1975, 52 (Suppl III): III-223-III-228.

40. Wyndham, C.R.C. Role of invasive electrophysiologic testing in the management of life-threatening ventricular arrhythmias. Am J Cardiol 1988, 62: 13I-17I.

41. Saini, V., Graboys, T.B., Towne, V. \& Lown, B. Reproducibility of exercise-induced ventricular tachycardia in patients undergoing evaluation for malignant ventricular arrhythmia. Am J Cardiol 1989, 63: 697-701.

42. Podrid, P.J., Venditti, F.J., Levine, P.A. \& Klein, M.D. The role of exercise testing in evaluation of arrhythmias. $A m J$ Cardiol 1988, 62: 24H-33H

43. Kim, S.G., Aboaf, A.P., Roth, J., Ferrick, K. \& Fisher, J.D. Prognosis of patients with ventricular tachycardia or fibrillation and a normal electrophysiologic study. Am Heart J 1991, 121: $77-80$.

44. Swerdlow, C.D., Freedman, R.A. \& Peterson, J. Determinants of prognosis in ventricular tachyarrhythmia patients without induced sustained arrhythmias. Am Heart J 1986, 111: $433-438$

45. Ruskin, J.N., Dimarco, J.P. \& Garan, H. Out of hospital cardiac arrest: electrophysiological observations and selection of long-term antiarrhythmic therapy. $N$ Engl $J$ Med 1980, 303: 607-613.

46. Wilber, D.J., Olshansky, B., Moran, J.F. \& Scanlon, P.J. Electrophysiological testing and non-sustained ventricular tachycardia. Use and limitations on patients with coronary artery disease and impaired left ventricular function. Circulation 1990, 82: 350-358

47. Brembilla-Perrot, B., Donetti, J., Terrier de la Chaise, A., Sadoul, N., Aliot, E. \& Juillière, Y. Diagnostic value of ventricular stimulation in patients with idiopathic dilated cardiomyopathy. Am Heart J 1991, 121: 1124-1131. 
48. Cardiac Arrhythmia Suppression Trial (CAST) Investigators. Preliminary report: effect of encainide and flecainide on mortality in a randomised trial of antiarrhythmia suppression after myocardial infarction. $N$ Engl J Med 1989, 321: 406-412.

49. Moosvi, A.R., Goldstein, S., Van der Brug Medendorp, S. et al. Effect of empiric antiarrhythmic therapy in resuscitated out-of-hospital cardiac arrest victims with coronary artery disease. Am J Cardiol 1990, 65: 1192-1197.

50. Stanton, M.S., Prystowsky, E.N., Fineberg, N.S., Miles, W.M., Zipes, D.P. \& Heger, J.J. Arrhythmogenic effects of antiarrhythmic drugs: a study of 506 patients treated for ventricular tachycardia or fibrillation. JACC 1989, 14: 209-215.

51. Burkart, F., Pfisterer, M., Koiwski, W., Follath, F. \& Burkhardt, D. Effect of antiarrhythmic therapy on mortality in survivors of myocardial infarction with asymptomatic complex ventricular arrhythmias: Basel Antiarrhythmic Study of Infarct Survival (BASIS). JACC 1990, 16: 1711-1718.

52. Morganroth, J., Anderson, J.L. \& Gentzkow, G.D. Classification by type of ventricular arrhythmia predicts frequency of adverse cardiac events from flecainide. JACC 1986, 8: 607-615.

53. Minardo, J.D., Heger, J.J., Miles, W.M., Zipes, D.P. \& Prystowsky, E.N. Clinical characteristics of patients with ventricular fibrillation during antiarrhythmic drug therapy. N Engl J Med 1988, 319: 257-262.

54. Pratt, C.M., Eaton, T., Francis, M.F. et al. The inverse relationship between baseline left ventricular ejection fraction and outcome of antiarrhythmic therapy: a dangerous imbalance in the risk-benefit ratio. Am Heart $J$ 1989, 118: $433-440$.

55. Torres, V., Flowers, D. \& Somberg, J.C. The arrhythmogenicity of antiarrhythmic agents. Am Heart J 1985, 109: 1090-1097.

56. Velebit, V., Podrid, P., Lown, B., Cohen, B.H. \& Graboys, T.B. Aggravation and provocation of ventricular arrhythmias by antiarrhythmic drugs. Circulation 1982, 65: 886-894.

57. Kuchar, D.L., Garan, H., Venditti, F.J. et al. Usefulness of sotalol on suppressing ventricular tachycardia or ventricular fibrillation in patients with healed myocardial infarcts. Am J Cardiol 1989, 64: 33-36.

58. Amiodarone vs Sotalol Study Group. Multicentre randomised trial of sotalol $v s$ amiodarone for chronic malignant ventricular arrhythmias. Eur Heart J 1989, 10: 685-695.

59. Brodsky, M.A., Allen, B.J., Luckett, C.R., Caparelli, E.V., Wolff, L.J. \& Henry, W.L. Antiarrhythmic efficacy of solitary beta-adrenergic blockade for patients with sustained ventricular arrhythmias. Am Heart J 1989, 118: 272-280.
60. DiCarlo, L.A.J., Susser, F. \& Winston, S.A. The role of beta-blockade therapy for ventricular tachycardia induced with isoproterenol: a prospective analysis. Am Heart J 1990, 120: $1347-1355$.

61. Fournier, C., Brunet, M., Bah, M. et al. Comparison of the efficacy of propranolol and amiodarone in suppressing ventricular arrhythmias following myocardial infarction. Eur Heart J 1989, 10: 1090-1100.

62. Hirsowitz, G., Podrid, P.J., Lampert, S., Stein, J. \& Lown, B. The role of $\beta$-blocking agents as adjunct therapy to membrane-stabilising drugs in malignant arrhythmia. $A m$ Heart $J$ 1986, 111: 852-860.

63. Myerburg, R.J., Kessler, K.M., Cox, M.M. et al. Reversal of proarrhythmic effects of flecainide acetate and encainide hydrochloride by propranolol. Circulation 1989, 80: 1571-1579.

64. ISIS-1 Study Group. Randomised trial of intravenous atenolol among 16027 cases of suspected acute myocardial infarction: ISIS-1. Lancet 1986, ii: 57-65.

65. Cox, J.L. Ventricular tachycardia surgery: a review of the first decade and a suggested contemporary approach. Sem Thorac Cardiovasc Surg 1989, 1: 47-103.

66. Hargrove, W.C. \& Miller, J.M. Risk stratification and management of patients with recurrent ventricular tachycardia and other malignant ventricular arrhythmias. Circulation 1989, 79 (Suppl I): I-178-I-181.

67. Scheinman, M.M. Catheter ablation: present role and projected impact on health care for patients with cardiac arrhythmia. Circulation 1991, 83: 1489-1498.

68. Evans, G.T., Scheinman, M.M., Zipes, D.P. et al. The percutaneous cardiac mapping and ablation registry: final summary of results. Pace 1988, 11: 1621-1626.

69. Ward, D.E. \& Camm, A.J. The current status of ablation of cardiac conduction tissue and ectopic myocardial foci by transvenous electrical discharges. Clin Cardiol 1986, 9: 237-244.

70. Landymore, R.W., Gardner, M.A., McIntyre, A.J. \& Barker, R.A. Surgical intervention for drug-resistant ventricular tachycardia. JACC 1990, 16: 37-41.

71. Hargrove, W.C., Josephson, M.E., Marchlinski, F.E. \& Miller, J.M. Surgical decisions in the management of sudden cardiac death and malignant ventricular arrhythmias: subendocardial resection, the automatic internal defibrillator or both. J Thorac Cardiovasc Surg 1989, 97: 923-928.

72. Manolis, S., Rastegar, H. \& Estes, N.A.M. Automatic implantable defibrillation: current status. JAMA 1989, 262: $1362-1368$.

73. Winkle, R.A., Mead, R.H., Ruder, M.A. et al. Long-term outcome with the automatic implantable cardioverter defibrillator. JACC 1989, 13: 1353-1361. 